\title{
REAL INTERPOLATION WITH A FUNCTION PARAMETER FOR MARTINGALE HARDY-LORENTZ AND BMO SPACES
}

\author{
REN YANBO
}

Abstract. This paper is devoted to the study of real interpolation between martingale HardyLorentz and BMO spaces in the framework of interpolation with a function parameter. We first establish some inequalities for the sharp functions of martingales. With the aid of these inequalities, some new interpolation theorems which generalize some fundamental interpolation theorems in classical martingale $H_{p}$ theory are proved. In particular, we show that

$$
\left(H_{p_{0}, q_{0}}^{s}, B M O_{2}\right)_{\rho, q}=\Lambda_{q}^{s}\left(t^{\frac{1}{p_{0}}} / \rho\left(t^{\frac{1}{p_{0}}}\right)\right),
$$

where $0<p_{0}<\infty, 0<q_{0}, q \leqslant \infty$ and $\rho \in Q(0,1)$.

Mathematics subject classification (2010): 60G42, 60G46.

Keywords and phrases: Martingale space, Hardy-Lorentz space, BMO space, real interpolation, function parameter.

\section{REFERENCES}

[1] R. HANKS, Interpolation by the real method between BMO, $L^{\alpha}(0<\alpha<\infty)$ and $H^{\alpha}(0<\alpha<\infty)$, Indiana Univ. Math. J., 26, 679-689 (1977).

[2] C. Bennet, R. Sharply, Interpolation of Operators, Academic Press, Boston, 1988.

[3] F. WeIsZ, Martingale Hardy Spaces and their Applications in Fourier Analysis, Lecture Notes in Math, Vol. 1568, Springer-Verlag, Berlin, 1994.

[4] J.L. Lions, J. Peetre, Sur une classe d'espances d'interpolation, Inst. Hautes Etudes Sci. Publ. Math., 19, 5-68 (1964).

[5] T. F. Kalugina, Interpolation of Banach spaces with a functional parameter. The reiteration theorem, Vestnik Moskov. Univ. Ser. I, Math. Meh., 30 (6), 68-77 (1975).

[6] J. Gustavsson, A function parameter in connection with interpolation of Banach spaces, Math Scand., 42, 289-305 (1978).

[7] S. Janson, Minimal and maximal methods of interpolation, J. Funct. Anal., 44, $50-73$ (1981).

[8] C. MeruCCI, Applications of interpolation with a function parameter to Lorentz, Sobolev and Besov spaces, Lecture Note in Math. 1070, 183-201 (1984).

[9] L. E. PERSSON, Interpolation with a parameter function, Math Scand., 59, 199-222 (1986).

[10] H. P. HeINIG, Interpolation of quasi-normed spaces involving weights, Can. Math. Soc., Conf. Proc., 1, 245-267 (1981).

[11] J. SoRIA, Tent spaces based on weighted Lorentz spaces, Carleson measures, Phd thesis, Washington University, 1990.

[12] T. HolmstedT, Interpolation of quasi-normed spaces, Math Scand. 26, 177-199 (1970).

[13] J. Bergh, J. LÖFström, Interpolation Spaces, Springer-Verlag, Berlin, 1976.

[14] D. L. BOURKHOLDER, R. L. GUNDY, Extrapolation and interpolation of quasi-linear operators on martingales, Acta Math., 124, 249-304 (1970).

[15] R. J. BagBy, D. S. KurTZ, A rearranged good $\lambda$-inequality, Trans. Amer. Math. Soc. 293, 71-81 (1986).

[16] R. L. Long, Martingale spaces and inequalities, Peking University Press, Beijing, 1993. 
[17] Y. L. Hou, Y. B. REN, Weak martingale Hardy spaces and atomic decompositions, Science in China, Series A Math., 49, 912-921 (2006).

[18] P. D. LiU, Y. L. HoU, M. F. WANG, Weak Orlicz space and its applications to the martingale theory, Sci China Math., 53, 905-916 (2010).

[19] Y. Jiao, W. Chen, P. D. LiU, Interpolation on weak martingale Hardy space, Acta Math. Sinica, English Series, 25 (8), 1297-1304 (2009).

[20] Y. JiaO, L. H. Peng, P. D. LiU, Interpolation for weak Orlicz spaces with $M_{\Delta}$ condition, Science in China. Ser. A, 51 (11), 2072-2080 (2008).

[21] Y. JiAO, P. D. LiU, L. H. PENG, Interpolation for martingale hardy spaces over weighted measure spaces, Acta Math. Hungar, 120 (1-2), 127-139 (2008).

[22] L. P. FAN, Y. JiAO, P. D. LIU, Lorentz martingale spaces and interpolation, Acta Mathematica Scientia, 30 B(4) 1143-1153 (2010).

[23] Y. B. REN, T. X. GuO, Interpolation of Lorentz martingale spaces, Sci China Math., 55, 1951-1959 (2012).

[24] Y. B. REN, T. X. GuO, Real interpolation between martingale Hardy and BMO spaces, Acta Math. Sinica, English Series, 29 (1), 65-74 (2013). 